auriculatum hns shown that it is not identical with solasonine.

Thanks are duo to the Chemical Society and tho Australian and New Zealand Association for the Advancement of Science for grants.

Auckland University College,

L. H. Briggs.

Auckland, New Zealand.

Juno 1.

1 Barger and Fraenkel-Conrat, J. Chcm. Soc., 1537 (1936).

Oddo and Caronna, Ber., 67, 46 (1931) and carlier papers.

2 Rochclmcyer, Areh. Pharm., 275, 330 (1937).

- Rochelmever, Arch. Pharm., 274, 513 (1930).

'Clemo, Jorgan and Raper, J. Chem. Soc., 1299 (1936).

- Tutin and Clewer, J. Chem. Soc., 105, 565 (1014).

' Levi, J. Soc. Chem. Ind., 49, $39 j \mathrm{~J}$ (1930).

Anderson and Briggs, J. Chem. Soc., 1036 (103i).

\section{Mechanism of Hydrolysis of Inorganic Esters}

$\mathrm{IBY}$ the use of water containing an excess over the normal of the heavy oxygen isotopo $\left({ }^{18} \mathrm{O}\right)$, it has been definitely and very directly shown that during the hydrolysis of the esters of weak carboxylic acids the brenk occurs at the $\mathrm{C}-\mathrm{O}$ bond of the ester, thus :

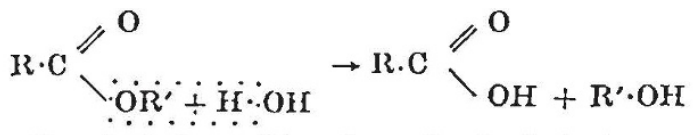

Thus tho alcohol resulting from the hydrolysis contains only the normal proportion of heavy oxygen isotope. This has been shown by Polanyi and Szabo' for the caso of alkaline hydrolysis and by Datta, Day and Ingold ${ }^{2}$ for tho case of acid hydrolysis after Roberts and Uroy ${ }^{3}$ had shown that the same $\mathrm{C}-\mathrm{O}$ bond is involved in the reverso process of esterifica. tion.

Wo have recently carried out a similar experiment on the alkaline hydrolysis of trimethyl phosphate, using aqueous sodium hydroxido prepared by acting with sodiun on water enriched in tho heavy oxygen isotopo. The hydrolysis was carried out at $80-90^{\circ} \mathrm{C}$. and proceeded until slightly more than one methyl group was removed. Tho resulting aqueous methyl alcohol was distilled off and purified by fractionation. Tho alcohol vapour wrs then passed over a red-hot platinum filament, whereby it was 'cracked' to givo carbon monoxide and hydrogen. 'The mixture, plus excess hydrogen, was passed over a nickel catalyst and tho carbon monoxido thereby reduced to water. This water thus contained the oxygen for the alcohol produced in the hydrolysis. Its density was measured, after purification, by the usual micro-pyknometer methods.

The results aro shown in the accompanying table, where $\Delta p$ (initial) is the excess density of the originnl water in parts per million and $\Delta p$ (final) is that of the water produced from the alcohol.

$\begin{array}{lc}\Delta p \text { (initial) } & \Delta \rho \text { (final) } \\ 180 & 0 \\ 180 & +1 \\ 200 & 0\end{array}$

Tho results show quite clearly that during tho hydrolysis the $\mathrm{P}-\mathrm{O}$ bond of the ester is broken thus :

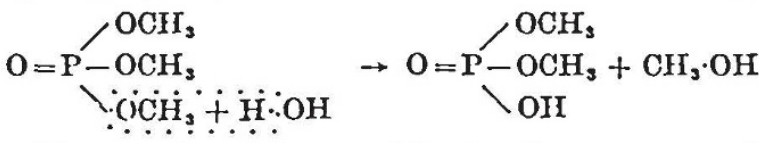

The experimental error of the density measurement may bo taken as $\pm \tilde{5}$ parts per million, so that at least 97 per cent of tho hydrolysis must proceed as indicated above.

This is a somowhat surprising result in viow of the fact that trimethyl phosphato is a fairly efficient methylating agents.

Density measurements of the residual wator show that, npart from the hydrolytic replacement of oxygen, there is only a very slight exchange of oxygen between the water and the ester.

During tho hydrolysis carried out as described above, a considerablo quantity of dimethyl ether is produced. Wo aro endeavouring to determino whether or not this ether contains excess of ${ }^{18} \mathrm{O}$, and also to investigate the course of the hydrolysis in acid solution. Wo also hopo to extend tho work to other inorganic esters.
Tho University,
IIanchester.
J. J3. M. Henbert.

June 30.

'Polanyi and Szabo, Trans. Far. Soc., 30, 509 (193ł).

2 Datta, J)isy and Ingold, J. Chem. Suc., 833 (1939).

- Urey and Roberts, J. Amer. Chem. Soc., 60, 2393 (19.3s).

- Gilflllan aud l'olanyi, Z. phys. Chem., 186, $25+$ (1033).

- Noller and Dutton, J. Amer. Chem. Soe., 55, $42+$ (1933).

\section{Application of the Coincidence Method to Testing} the Lifetime and Level Scheme of Radium C'

'Tus: application of the coincidence method to investigations of nuclear processes may bo extended in certain ways by using a variablo resolving time of the coincidenco circuit and by measuring pseudo. coincidences, that is, counts arising from two impulses which aro not simultaneous but separated by a very short time. The number of 'coincidences' given by two particles, in which ono follows the second in $\Omega$ timo determined by the radioactive constant $\lambda$, is proportional to $1-e^{-\lambda x}$, being the length of the impulso. Tho duration of tho impulso is dependent upon the electrical constants of the circuit, and by changing tho constants of ono or both circuits it is possible to obtain various determined lengths of the impulses. In order to mensure $\lambda$, two sets of experiments aro made: (a) with the impulso $\tau_{1}$ of the first particle long and of different durations, and the impulse $\tau_{2}$ of the second very short; $(b)$ with the conditions roversed. The differenco $a-b$ is equal to $\frac{N_{1} N_{2}}{N}\left(e^{-\lambda_{1}}-e^{-\lambda r_{1}}\right)$ whero $N_{1}$ and $N_{2}$ aro tho number of counts in each counter, $N$ the number of disintegrations. The method of lengthening the impulses only from ono kind of particlo allows ono also to decide tho order in which the particles aro emitted.

In these experiments tho method was applied to the transformation

$$
\mathrm{RaC} \stackrel{\beta}{\longrightarrow} \mathrm{RaC}^{\prime} \stackrel{\alpha}{\longrightarrow} \mathrm{RaD} \text {. }
$$

Coincidences wero observed between the various types of radiations, namely, $\alpha-\beta, \alpha-\gamma, \beta-\gamma$, and $\gamma-\gamma$. The $\beta$ - and $\gamma$-radiations were measured in the usual wny by means of Geiger-yliuller tubes; tho $\alpha$-particles wero counted separately by operating a Geiger-Miiller tube in the proportional region. The curve on the accompanying graph gives the number of real 'coincidences' $(a-b)$ from radium $\mathbf{C} \beta$-particles and radium $C^{\prime} \alpha$-particles as $\Omega$ function of the length of the impulso from the $\beta$-particles. From this curve one obtains a value for the half-lifetime of radium $\mathrm{C}^{\prime}$ of $1.40 \pm 0.15 \times 10^{-1}$ sec.*

The investigation of the $\alpha-\gamma$ coincidences gives $\Omega$ curve which is identical with tho former, showing 